\title{
Fazit, Ausblick und Nachwort
}

\section{Das normative Paradoxon}

Die normative Prämisse des Gleichheitsanspruchs und das Versprechen der Reduktion von Ungleichheit treiben die demokratischen Gesellschaften Europas dynamisch an. Als zentraler Ausgangspunkt der "großen Gereiztheit« wurde im vorliegenden Buch das normative Paradoxon genannt, das entsteht, wenn es einen Widerspruch zwischen zentralen Versprechen der pluralen Demokratie - nämlich Anerkennung, Chancengleichheit und Teilhabe für alle Bürger*innen - und der empirischen Realität gibt, die nachweisbar von sozialer Ungleichheit und Anerkennungsdefiziten gekennzeichnet ist, und zwar speziell, wenn es um die strukturelle, soziale, kulturelle und identifikative Anerkennung nicht-dominanter Gruppen geht. Nun könnte man gelassen zurückblicken und argumentieren, dass sich dieses normative Paradoxon demokratietheoretisch und -geschichtlich letztlich unvermeidlich durch unsere modernen Gesellschaften zieht: Bereits Tocqueville hat dieses Paradoxon beschrieben und weitere Demokratietheoretiker haben es immer wieder bestätigt. So what?

Zeitgeschichtlich neu ist, dass sich das Wissen um das Recht auf Gleichheit und Anerkennung im Gegensatz zu Tocquevilles Zeiten erhöht hat. Nicht nur das: Dieses Recht wurde regelrecht zur politischen Norm par excellence - quasi zum Anrecht - ganz so, als wollte die »freie Welt« nach dem Zusammenbruch der bipolaren Weltordnung klar machen, dass sie nicht nur ökonomisch überlegen war, sondern auch den normativen Anspruch des Kommunismus - nämlich die Gleichheit aller Bürger"innen - besser umsetzen kann. Gleichzeitig hat sich allerdings durch zahlreiche Mess-, Erfassungs- und Statistikverfahren das empirische Wissen um die Ungleichheit erhöht. Die gesellschaftliche Ungleichheit, die sich sowohl ökonomisch als auch politisch und sym- 
bolisch artikulieren kann, ist also nicht mehr nur ein Gefühl, was schon Treiber genug sein kann - sie ist eine überprüfbare Gewissheit, die sich an einem sehr hoch aufgehängten normativen Versprechen misst. Durch gesteigerte Information wird die Erkenntnis der bestehenden Ungleichheit salienter, fällt mehr ins Auge und wird stärker moralisch geächtet.

Dass dieses Versprechen der Gleichheit in der Bevölkerung so stark auf eine Anspruchshaltung der Gewährung trifft, liegt auch daran, dass die meisten europäischen Demokratien in den letzten Jahrzehnten tendenziell liberal ausgerichtet waren. Selbst wenn sie konservativ regiert wurden, war der Orientierungsgedanke der Regierungen ihren Bevölkerungen gegenüber zumeist noch liberal gerahmt und der Gleichheitsgrundsatz wurde als sinnstiftender Endpunkt der Politik und quasi als moralisches Leitmotiv formuliert, an dem sich die Regierungen messen lassen mussten. Selbst wenn dieser Gleichheitsgedanke durch eine zunehmend neoliberale Wirtschaftsordnung nach dem Ende des Ost-West-Konfliktes konterkariert wurde, so blieb er doch als Leitmotiv weitererzählt. Die hohe Norm wurde spätestens mit Beginn der Finanzkrise 2008 als unerfüllbar erkannt und seit dieser Zeit ist das rechte und rechtspopulistische Angebot, die Norm zu senken bzw. sie auf die eigene ethnische »Stammbevölkerung « einzuschränken, wieder zu einem ernsthaften konkurrierenden Angebot in europäischen Gesellschaften geworden. Zwar waren rechte und rechtspopulistische Parteien schon vor der Finanzkrise in Europa aktiv und organisiert, aber nach der Finanzkrise hat sich die Zahl der rechtspopulistischen Parteien in den Parlamenten Europas mehr als verdoppelt und der Anteil rechtspopulistischer Parteien an der Sitzverteilung liegt in Ländern wie Polen und Ungarn bei über $50 \%$; in Finnland, Italien, Österreich oder der Schweiz sind rechtspopulistische Parteien an der Regierung beteiligt, in Großbritannien, Frankreich und Deutschland treiben sie die anderen Parteien vor sich her und verschärfen den Ton der politischen Debatten.

Dies wiederum ging zeitgleich mit einem Pluralitätserwachen in europäischen Gesellschaften einher, von denen viele seit der Jahrtausendwende eine Debatte um ihre nationale Identität führten, die sie vor dem Hintergrund zunehmender Diversität zu reformulieren begannen. Eine Gleichzeitigkeit von ungleichzeitigen Phänomenen prägt also die postmigrantischen Gesellschaften Europas, was auf ihre zunehmende Hybridisierung schließen lässt: Während sie ihre Migra- 
tionsrealität, die keineswegs neu war, politisch, rechtlich und symbolisch zu verhandeln begannen, während zunehmend mehr Menschen in diese Verhandlungen mit dem Anspruch der Anerkennung und >Gleichberechtigtheit eintraten, wuchsen parallel die Kenntnisse über die Anerkennungslücken. Und mit der Forderung einer Anerkennungsgleichheit wuchs im Schatten der nationalen Narrative, der neoliberalen Ungleichheitspolitik und der zunehmenden kognitiven Dissonanz die Macht der rechten Gegennarrative. Diese stellten die Frage danach, ob die Gleichheit, wenn sie denn schon so utopisch sei, nicht wenigstens nur dem eigenen Kollektiv zugesprochen werden sollte. Wenn die Norm nicht mehr von der Gleichheit aller ausgeht, dann ist das normative Paradoxon vielleicht auch nicht mehr so spannungsgeladen und dysfunktional.

Zur Erinnerung: Ausgangspunkt der Abschrift dieses Buches war eine beobachtbare Normverschiebung in europäischen Gesellschaften. Angelehnt an Durkheims Vorstellung einer Anomie war damit das Vergessen der zentralen Normen, die das kollektive Bewusstsein und Gedächtnis (conscience collective) einer Gesellschaft prägen, gemeint. Wenn die zentrale Norm der Gleichheit, die Europas Gesellschaften nach dem zweiten Weltkrieg antreibt und das kollektive Bewusstsein prägt, aufgrund seiner Nichterfüllbarkeit schlicht für manche Gruppen infrage gestellt oder nach hinten gestellt wird, dann führt dies in einen anomischen, spannungsgeladenen Zustand. Das normative Paradoxon von heute ist also nicht zu vergleichen mit dem normativen Paradoxon der Demokratiegeschichte, das hinnehmbar ist, weil es keine dysfunktionale Auswirkungen hat. Es ist nicht mehr nur ein demokratietheoretisch hinzunehmendes Konstrukt. Es ist »die Mutter aller Probleme« - solange der Anspruch auf Gleichheit als Leitmotiv moderner Demokratien Bestand hat.

Anhaltende oder sogar steigende Ungleichheit, wie sie in Europa seit der Finanzkrise 2008 in vielen Ländern zu beobachten ist, prallt also auf ein persistentes Selbstbild eines moralisch überlegenen Kontinents, der seine Identität an Kernnarrative von Aufklärung, Menschenrechten und Toleranz sowie an die emanzipativen Ideen der Freiheit und Gleichheit knüpft. Die Frage danach, ob wir wirklich sind, wer wir glauben zu sein, schleicht sich als permanente Verunsicherung in die kollektive und individuelle Selbstbeschreibung ein. Das erklärt die "große Gereiztheit«. Die Gesellschaft scheitert nicht an der Migration sondern post-migrantisch: am Umgang mit der Gleichheitsfrage, die 
durch die Migration pars pro toto gestellt wird. Der Kampf um gleiche Rechte, um Anerkennung, Chancengleichheit und Teilhabe wird heutzutage über die Migrationsfrage geführt. Die Migrationsfrage ist somit zur neuen sozialen Frage unserer Zeit geworden. Anhand der Migrationsfrage werden Fragen der Verteilungsgerechtigkeit, der Teilhabe und der kulturellen Selbstbeschreibung Europas exemplarisch verhandelt - am Umgang mit ihr wird der Umgang mit Pluralität und Differenz durchgespielt, am Scheitern an ihr der Grad der offenen Gesellschaft gemessen.

\section{Die Pluralität}

Was das normative Paradoxon der heutigen Zeit von der demokratietheoretischen und -historischen weiter unterscheidet, ist, dass zu den drei zentralen sich widersprechenden Prämissen, die in der Demokratie als paradoxal zueinander beschrieben werden - Gleichheit, Freiheit und Sicherheit -, eine intersektionale Frequenz hinzukommt: die Pluralität. Die Pluralität fordert die Gleichheit heraus, da sie beansprucht, nicht nur in der Gleichheit, sondern auch in der Differenz gleich behandelt zu werden (ausgedrückt z. B. im Hashtag \#vonhier). Sie fordert die Freiheit heraus, wo sie auch das Recht auf Unfreiheit als freie Entscheidung mit einbringt (z. B. im Anspruch, Kopftuch zu tragen), und sie fordert die Sicherheit heraus, z. B. dort, wo sie die Grenzen infrage stellt (z. B. im pluralen europäischen Schengenraum oder im Sinne der Hinterfragung von Geschlechtergrenzen). Die Pluralitätsfrage ist also mitnichten nur eine Frage der Migration. Die modernen Demokratien haben sich anhand vielfältiger Aspekte diversifiziert: Geschlechterverhältnisse, sexuelle Identitäten, religiöser Synkretismus, Multi- und Crosssprachlichkeit etc. Jedoch ist Migration $\mathrm{zu}$ einem diskursiv omnipräsenten Thema geworden und somit sehr salient. Die Frage des Umgangs mit Pluralität wird vor allem anhand der Migrationsfrage debattiert.

Dies erzeugt in der postmigrantischen Gesellschaft eine zusätzliche Spannung: (1) auf der einen Seite die oben genannte, zwischen dem Gleichheitsversprechen und der empirisch nachweisbaren Ungleichheit, die nicht nur, aber in hohem Maße Migrant"innen und ihre Nachkommen betrifft. (2) Parallel ist eine subjektbasierte Ambivalenz zu erkennen: Denn diese nicht-anerkannte Gruppe wächst stetig demo- 
graphisch weiter an und Migrant"innen und ihre Nachkommen beanspruchen nicht nur strukturelle und soziale Teilhabe, sondern darüber hinaus beanspruchen sie auch noch, Teil der kulturellen und nationalen Identität zu sein, also symbolische und emotionale Identifikationsangebote zu erhalten. Sie fordern dabei auch, ihre Differenz nicht ablegen zu müssen: Sie wollen also gleich und verschieden zugleich sein. Damit treiben sie die Anerkennungsforderungen noch weiter an und es kommt zu einem Umsetzungsstau in Bezug auf die Anerkennungsgewährung, was wiederum einen Spannungszustand in der Gesellschaft erzeugt und teilweise mit einer Senkung der demokratischen Normen - also der Anrechte von Migrant"innen und ihren Nachkommen auf gleichberechtigte Anerkennung - beantwortet wird. Eine gleichberechtigte Anerkennung bedeutet auch eine veränderte Politik, die Anerkennungsziele definiert und diese an Quoten oder Paritätsvorstellungen austariert. Dies kann dazu führen, dass die eigene privilegierte Position in der Gesellschaft zugunsten einer aktiven Gleichstellungspolitik in Frage gestellt wird. Wenn Frauen die gleiche Anzahl an Sitzen in Parlamenten erhalten sollen, dann heißt dies zwangsläufig, dass weniger Männer dort Platz haben werden. Und wenn eine Quote für die Beteiligung von Migrant"innen und ihren Nachkommen eingefordert werden sollte, um Repräsentationslücken zu schließen, dann würde das bedeuten, dass dafür Platz geschaffen werden müsste, was zu Ungunsten nicht-migrantischer Deutscher gehen würde etc.

Um die Nichtanerkennung zu legitimieren, werden Migrant*innen und ihre Nachkommen von einigen Teilen der Gesellschaft daher pauschal zu Fremden erklärt, die außerhalb stehen und demnach in ihren Anspruchspositionen ignoriert oder zurückgewiesen werden dürfen oder sogar müssten. Dadurch wird wieder klar, wer zu »uns« gehört und wer nicht und wem der Anspruch auf Gleichheit zusteht und wem nicht. Klassifizierungsschemata erlangen dadurch wieder ihre Gültigkeit, nachdem sie zunehmend durch die Postmoderne, die liberale, plurale Demokratie und die Hybridisierung der Gesellschaft infrage gestellt worden waren. »Der Islam ist ein Fremdkörper in Deutschland«, betont Alexander Gauland, der Vorsitzende der rechtspopulistischen AfD in Deutschland immer wieder, um legitime Anerkennungsanforderungen von Muslimen in Deutschland abzuwehren. Dies geschieht mehr als zehn Jahre, nachdem die erste Deutsche Islam Konferenz im Jahr 2006 explizit von Seiten der Bundesregierung das Signal an die Muslime gesendet hat, dass sie und ihre Religion Teil dieses Landes 
sind. Simmel hat dieses ambivalente Moment der Abwehr bereits 1908 beschrieben: »Es ist hier also der Fremde nicht [...] der Wandernde, der heute kommt und morgen geht, sondern [...] der, der heute kommt und morgen bleibt.« (Simmel 1908: 509) Die Hybridität, die sich durch die, die vormals eingewandert und heute Teil der Gesellschaft geworden sind - die also einmal Wandernde waren und heute Einheimische $\mathrm{zu}$ sein beanspruchen -, einstellt, löst die etablierten Grenzen der Zugehörigkeit auf und stellt die einstmalige Sicherheit, zu wissen, wer dazugehört und wer nicht, wer Deutscher ist und wer nicht, infrage. Die Grenzen zwischen dem Fremden und dem Eigenen verschwimmen. Und während sie sich über die Generationen hinweg zunehmend auflösen, wächst das Unbehagen innerhalb jenes Teiles der Bevölkerung, für den die Pluralität eine wachsende Unordnung und Infragestellung erlernter Codes darstellt.

Zygmunt Bauman hat die Konstruktion von Fremdheit als einen Effekt der Moderne bezeichnet, weil die Moderne mit ihrer Idee der kulturellen Einheit und dem Bestreben, (gesellschaftliche) Ordnung auf der Basis binärer Gegensätze zu konstruieren, die Kategorien des Eigenen und des Fremden zunehmend manifestiert hat (Bauman 1999). Die pmG ist aber eine Gesellschaft, in der etablierte binäre Codierungen entlang kultureller, ethnischer, religiöser und nationaler Grenzziehungen verschwimmen. Aber auch die Grenzen der Generationen, Geschlechter, Nationen oder Links-rechts-Positionierung werden brüchig. Die etablierten cleavages von rechts versus links, jung versus alt, religiös versus säkular oder städtisch versus ländlich werden entlang der Migrationsfrage brüchig. Es wird unklar, welches Lager pro und welches gegen Migration ist: ausländerfeindliche Konservative oder religionsfeindliche Linke gibt es ebenso, wie die große Willkommenskultur im langen Sommer der Migration gerade auch in konservativen, ländlichen und christlichen Regionen hoch war. Und ebenso wie die ungebrochene Solidarität langjähriger Allianzpartner*innen im liberalen und linken Spektrum der Gesellschaft weiterhin besteht, bricht parallel in Teilen des Altfeminismus eine neue Front gegen Migration auf. Umgekehrt zeigen sich viele ältere Menschen, die noch den Krieg und die Flucht erlebt haben, empathischer und offener als junge Identitäre, die keine Kriegsangst mehr kennen, die sie demokratisch bindet, und die die liberalen Errungenschaften der Achtundsechziger belächeln oder die Europa-Konstruktion nur als institutionelle Fehlkonstruktion betrachten und nicht als normatives Gefüge des Aufeinanderzu- 
gehens. Gleichzeitig sind viele Junge kosmopolitischer und bereits in einem Deutschland aufgewachsen, das sich als Einwanderungsland erzählt, was sie offener für Migration macht, während ältere Menschen sich nach einem "alten Deutschland« sehnen, welches es nicht mehr gibt und die Migrant"innen dafür verantwortlich machen. Die postmigrantische Gesellschaft ist ambivalent. Wer ist hier Alliierter und wer Antagonist? Postmigrantische Allianzen kommen in der Aushandlung der Anerkennung von Gleichheitsgrundsätzen zusammen und sind mit antagonistischen Akteuren und Positionen konfrontiert, die nicht nur die Allianzen und ihre Aushandlungen bekämpfen, sondern die Prämisse ihrer Zusammenkunft selbst infrage stellen, nämlich das Recht auf Anerkennung, Gleichheit und Teilhabe als ein universelles Versprechen.

\section{Ausblick:}

\section{Ein neues Narrativ für die postmigrantische Gesellschaft}

Migration ist in der postmigrantischen Gesellschaft ein Bezugspunkt der Ambivalenz: Sie ist Normalität und Notstand gleichermaßen; Migration wird gleichzeitig politisiert und popularisiert. Migration ist das Dauerthema - warum denn dann post-migrantisch?

In Deutschland leben im Jahr 2019 knapp 20 Millionen Menschen mit einem sogenannten Migrationshintergrund, von denen bereits zehn Millionen die deutsche Staatsangehörigkeit besitzen (Statistisches Bundesamt 2018a). Knapp 40\% aller schulpflichtigen Kinder in diesem Land haben eine Migrationsgeschichte (Statistisches Bundesamt 2018c) - in absehbarer Zeit werden sie wahlberechtigt sein und über die politischen und gesellschaftlichen Geschicke mitentscheiden. Dennoch ist es bis dato nicht gelungen, aus dieser Tatsache eine Erzählung zu generieren, die eine neue, plurale und migrationsoffene nationale Identität formuliert, die sich dann mittelfristig in ein kollektives Gedächtnis einspeist und über ein politisches Bekenntnis hinauswirkt. Allerdings: In einer postmigrantischen Gesellschaft werden narrative Neudeutungen und eine Verknüpfung der Geschichten der Migration mit der Erzählung der deutschen Identität verlangt und eingefordert, wie exemplarisch das Buch »Ich bin von hier. Hört auf zu fragen« von Ferda Ataman (2019) zeigt.

Narrative werden stets diskursiv konstruiert und passen sich an die Gegenwart und neue Praxen der Gemeinschaftsbildung an. Sie werden 
immer wieder neu ausgehandelt. Im öffentlichen Raum stehen Deutungsmodelle nebeneinander, die um die Diskurshoheit konkurrieren. Narrative zur deutschen Identität beruhen noch immer überwiegend auf rekonstruktiven Bezügen - also auf der Vergangenheit. Dadurch wird die deutsche Identität als eine über die Zeit hinweg gewachsene Beziehung simuliert, welche in teils dramatischen Erfahrungsbezügen eine Gemeinschaft erzeugt hat, in die diejenigen nicht eintreten können, die diese Historizität nicht teilen. Diese rekonstruktiven Narrative sind häufig affektiv und pathosgeladen und werden nur dem eigenen Kollektiv zugestanden. Somit dominieren im öffentlichen Diskurs reduktionistische Ansätze, die das Deutschsein immer wieder als exklusive, nur für einige »Auserwählte« bestimmte Kategorie beschreiben.

Dabei könnte auch eine andere Erzählung angeboten werden, die durchaus glaubhaft ist: Dieses Land hat sich verändert. Deutschland normalisiert sich, indem es kulturell, ethnisch, religiös und national vielfältiger wird. Es war schon immer von Vielfalt geprägt. Es ist sozusagen aus einem Moment der Vielfalt entstanden. 1848 kamen in der Paulskirche vielfältige Fürstentümer zusammen, die sich mitnichten als gleich gefühlt haben. In Deutschland sprach man Polnisch, Sorbisch, Französisch, Russisch, wie im Kapitel zu pluralen Demokratien in diesem Buch gezeigt wurde. Deutschland hatte immer schon eine mittlere Kontinentallage. Man konnte nicht über dieses Land hinwegfliegen, man wanderte hindurch. Römer siedelten, Hunnen siedelten, selbst Syrer ließen sich als Bogenschützen in Bayern nieder. Pluralität ist ein Markenzeichen dieses Landes. Wir leben im Zeitalter der globalen Migration, das jenes der überschaubaren nationalen Entscheidungsprozesse abgelöst hat. Alles bewegt sich über Grenzen hinweg - Güter, Ideen, Menschen und Identitäten. Sie werden hybrider. Das erzeugt Unübersichtlichkeiten, denen Populisten mit dem Versprechen von Reduktion und Übersichtlichkeit entgegentreten. Dabei kann diese Reduktion sehr gewaltvoll vonstattengehen: allein von 2015 bis Anfang 2019 gab es über 8.000 dokumentierte Angriffe auf Asylbewerbende und ihre Unterkünfte in Deutschland. ${ }^{1}$ Das sind mehr als fünf Angriffe pro Tag.

Postmigrantische Gesellschaften sind also geprägt von Konflikten zwischen jenen, die unter Demokratie gleiche Rechte für alle Bürger*in-

1 Siehe hierzu die Chronik flüchtlingsfeindlicher Vorfälle der Amadeu Antonio Stiftung und des Vereins PRO ASYL: https://www.mut-gegen-rechte-gewalt.de/service/ chronik-vorfaelle. 
nen verstehen, und jenen, die Vorrechte nur für die jeweils eigene Gruppe beanspruchen. Wir beobachten hier zunehmend eine Spannung zwischen kognitiver und affektiver Wahrnehmung von Zusammenleben: Während auf der einen Seite eine Gelassenheit und Akzeptanz von Migration zu beobachten ist, sind Abwehrhaltungen und Stereotype gegenüber sichtbaren Minderheiten auf einem hohen Niveau stabil. Auch gegenüber snicht sichtbaren< Minderheiten wie (säkularen) Juden oder Homosexuellen hält sich eine latente emotionale Abwehr. Man könnte geneigt sein zu denken: Solange sie sich »keinen Schaden zufügen «, sollen die Menschen doch denken, was sie wollen - jeder hat ein Recht auf Antipathie, wir sind nicht verpflichtet, einander zu lieben! Aber die letzten Jahre haben deutliche Gewaltanstiege dokumentiert und wir müssen uns fragen, ob demokratische Grundwerte wie Gleichheit und Gleichwertigkeit als purer Wohlfühldiskurs implodieren, wenn ihre Erfüllung nicht in aktiven Allianzen für alle erstritten, sondern überrumpelt wird von dem berauschenden Angebot einer überschaubaren Volksgemeinschaft - die durch die Rechtspopulisten als Alternative zum verwirrenden »anything goes « der Postmoderne angeboten wird. Wir müssen also die Frage stellen, ob plurale Demokratien an ihrer Pluralität zugrunde gehen können, wenn sie nicht durch ein sinnstiftendes Narrativ zusammengehalten werden.

Die Frage ist also, ob heterogene Gesellschaften nicht einer übergeordneten politik- und handlungsleitenden Großerzählung bedürfen, die sie in ihrem politischen und narrativen Selbstbild strukturiert, weil die Pluralität als pures Nebeneinander nicht als sinngebend empfunden wird, wenn ihr kein sinnstiftender Endpunkt vorausgeht, auf den diese Vielheit zuläuft. Pluralität als einfache empirische Kategorie ist kein hinreichend starker Treiber für Akzeptanz - vor allem, weil diese Pluralität, die durchaus auch als positiv und herausfordernd betrachtet wird, oftmals als bedrohlich, verunsichernd und überfordernd wahrgenommen wird und dieses Gefühl von rechten Populisten und Islamisten instrumentalisiert wird, indem permanent Angebote zur Ambiguitätsreduktion gemacht werden. Die Angebotsunterbreiter der AfD, der Brexiteers oder des Rassemblement National warnen vor Parallelstrukturen, Chaos, Unordnung und Bezugslosigkeit, wo andere Mehrfachzugehörigkeiten, Fluidität und Code-Switching hochhalten. Die Salafisten und Islamisten winken mit »wahren« Identitäten, während die andere Seite die Unentschiedenheit und die Hybridität als Normalität betrachtet. 
Die große Frage, die sich nun an die postmigrantischen Alliierten für ihre zukünftige Positionierung und Verteidigung der pluralen Demokratie stellt, ist, ob es auch Großerzählungen geben kann, die sinnstiftend und strukturierend auf Politik und Zivilgesellschaft Einfluss nehmen und gleichzeitig die Polarisierung überwinden. Dabei stellt sich wieder einmal die Frage, ob ein Verfassungspatriotismus als Basis ausreicht, um heterogene Gesellschaften - etwa die deutsche Gesellschaft - zukünftig politisch weiterzudenken. Braucht es vielleicht zusätzlich einen sinnstiftenden Endpunkt, ein handlungsleitendes Motiv oder Leitbild, welches politisch definiert, wie dieses neue plurale Deutschland sich erzählt und, vor allem, wie es plant, diese Erzählung zu erfüllen? Wer erzählt und entwirft dieses Motiv? Und lässt ein solches Leitbild - eine Neudefinition des pluralen postmigrantischen Deutschlands - nicht auch wieder »Andere« zurück, wie Pegida und AfD-Anhänger*innen oder Salafist*innen?

Wenn die Sozialpsychologie sagt, dass es keine kollektive soziale Identität geben kann ohne die Existenz einer outgroup, dann müssen wir entweder Heterogenität mit dem Fortbestand von outgroups zusammendenken oder wir denken weniger sozialpsychologisch und orientieren uns am philosophischen Ansatz Martin Bubers (1995) und seinem >Ich< in Gegenüberstellung zum >Du<. So könnte ein neues deutsches ,Wir<, zwar immer noch nur in Kombination mit einem >Ihr gedacht werden, aber dieses >Ihr< wäre keine dichotome Ingroup-outgroup-Kategorie, sondern ein alliierter Bezugspunkt eines sinnstiftenden Narratives, auf das beide zustreben - in einer dynamischen Integrationslogik, die danach strebt, gemeinsam Ungleichheiten nicht nur für die eigene soziale Gruppe abzubauen, sondern die Analogien der Ungleichheit zu erkennen.

Gleichwertigkeit als Basis eines Zusammenlebens in einer Gesellschaft zu sehen, die von Pluralität gekennzeichnet ist, ist allerdings eine höchst normative Erzählung. Diese mit Forderungen nach ökonomischer und struktureller Gleichheit zu verknüpfen, müsste Aufgabe des Leitbildes sein. Die Integration in dieses Leitbild müsste als »Integration für Alle«, die keinen Zugang zu zentralen gesellschaftlichen Gütern und Ressourcen haben, definiert werden. Dies wäre dann eine Zielperspektive, die für die Politik handlungsleitend und für die Zivilgesellschaft sinnstiftend wirken könnte.

In den letzten Jahren gab es auf Demonstrationen und im öffentlichen Raum immer Plakate, auf denen stand: Deutschland ist bunt. Die- 
ses etwas einfältig klingende Narrativ birgt eine kraftvolle Erzählung. Es beschreibt eine empirische Realität, eine normative Grundlage und eine dialektische Positionierung zugleich: Wir verteidigen die plurale Demokratie gegen euch. Es muss gelingen, dieses Narrativ zu festigen und im kollektiven Gedächtnis zu verankern. Und parallel muss es gelingen, auch jene $\mathrm{zu}$ integrieren, denen das bunte Deutschland nicht gefällt. Für beides braucht es politische Maßnahmen auf dem Weg zu einer gleichberechtigten Gesellschaft. Das wäre eine postmigrantische Politik im Sinne eines gesamtgesellschaftlichen Ziels: Integration für alle.

\section{Nachwort: Ist Deutschland nun eine postmigrantische Gesellschaft? Eine didaktische Checkliste zum Abschluss}

Wenn Sie dieses Buch bis hierher gelesen haben und sich immer noch fragen, was eine postmigrantische Gesellschaft ist, oder wenn Sie nur Einleitung und Fazit gelesen haben, weil Sie wenig Zeit haben, dann soll dieses Nachwort Ihnen eine kleine Checkliste an die Hand geben, um das Konzept der postmigrantischen Gesellschaft dicht und kondensiert zu verinnerlichen.

Zunächst der Leitgedanke: In postmigrantischen Gesellschaften (pmG) geht es nicht mehr darum, ob ein Land sich als Einwanderungsland beschreibt, sondern wie das Einwanderungsland gestaltet wird.

Die pmG entsteht und formt sich nach der Migration - bzw. nach der Anerkennung der Migration als grundlegendem Bestandteil dieser Gesellschaft. Seitdem Deutschland politisch als ein Einwanderungsland bezeichnet wird, prägt die Aushandlung der Rechte und Pflichten der Migrant*innen den politischen Diskurs in diesem Land. Migration steht nicht mehr für das temporäre Kommen, sondern für das langfristige Bleiben, für das, was nach der Einwanderung passiert - für eine postmigrantische Neuordnung etablierter Strukturen, Prämissen, Privilegien und Perspektiven.

Migration entwickelt sich dadurch zu einer zentralen gesellschaftlichen Konfliktlinie. Fragen der Zugehörigkeit, des Ausschlusses und der Integration sind in der Lage, ganze Gesellschaften zu polarisieren und in nervöse Spannung zu versetzen. Die postmigrantische Gesellschaft ist geprägt von einer Omnipräsenz des Migrationsdiskurses, der andere zentrale Fragen der Ungleichheit verdeckt. Das »post-« ist hier 
kein zeitliches Präfix; es bedeutet, dass es in diesen Gesellschaften mittels neuer Allianzen zunehmend gelingt, hinter das Migrationsnarrativ zu schauen und Analogien der Ungleichheit zu erkennen.

Dabei ist eine der zentralen Forderungen, die die postmigrantischen Gesellschaften normativ aushandeln, die nach der Überwindung der binären Codierung der Gesellschaft in Einheimische und Zugewanderte. Die pmG löst die etablieren binären Codes durch eine zunehmende Hybridisierung der Gesellschaft auf und gelangt somit immer wieder über die Hierarchisierung in Zugehörige und Nichtzugehörige hinaus und irritiert damit etablierte Sprachpraxen, Interpretationsmuster und nationale Identitätszuschreibungen.

\section{Ist Deutschland nun eine postmigrantische Gesellschaft?}

Abschließend bleibt eine letzte zu klärende Frage: Steht die postmigrantische Gesellschaft für eine Utopie oder beschreibt sie einen IstZustand? Sprich: Ist Deutschland eine postmigrantische Gesellschaft oder soll sie erst zu einer werden? Zunächst: Ein »post-« kann niemals eine Utopie beschreiben; ein »post-« definiert einen Transit - weg von etwas Vorherigem -, aber implizit deutet es auch schon auf ein zukünftiges Ziel. Insofern wäre die Utopie wohl: die gleichberechtigte Gesellschaft. Von der ist Deutschland noch weit entfernt - aber in der postmigrantischen Gesellschaft wird der Blick für die bestehenden Paradoxien und Ungleichheiten geschärft, die aus dem Weg geräumt werden müssen, um der Utopie ein Stück näher zu kommen. Das ist schon einmal ein erster Schritt.

Dies können Sie für sich anhand der fünf zentralen Konstrukte, die in diesem Buch ausführlich beschrieben wurden, überprüfen. Die Konstrukte, die die Dynamik postmigrantischer Gesellschaften bestimmen, lauten: Anerkennung, Aushandlung, Allianz, Antagonismus und Ambivalenz. Sie wurden in diesem Buch theoretisch hergeleitet und empirisch anhand repräsentativer Datensätze überprüft. Folgende Fragen wurden dabei mit Bezug auf Deutschland gestellt:

1. Ist Migration eine Grundlage der gesellschaftlichen Selbstbeschreibung und gibt es eine politische Anerkennung dessen? Ja. Seit der SüssmuthKommission 2001 ist es politisch anerkannt, dass Deutschland ein Einwanderungsland ist. Mittlerweile wird dies von einem Großteil der Bevölkerung für die wirtschaftliche und kulturelle Entwicklung des Landes als positiv beschrieben. Bei der Frage, ob Migra- 
tion gut für das soziale Miteinander ist, stimmen zwar nur noch $54 \%$ der Bevölkerung zu (siehe Abb. 15), jedoch wird deutlich, dass die Mehrheit der Bevölkerung in Deutschland nicht mehr über die Frage des `obく, sondern über die Frage des `wie`streitet - ein klarer Indikator für eine postmigrantische Gesellschaft.

2. Wurde die Aushandlung der Anerkennung von Gleichheit und Teilhabe auf rechtlicher, struktureller und symbolischer Ebene seitdem verstärkt? Ja. Das kann exemplarisch anhand von Gesetzgebungsprozessen zur doppelten Staatsangehörigkeit, zum Zuwanderungsrecht, zur Anerkennung ausländischer Abschlüsse usw. nachvollzogen werden. Auch Bildungsanstiege oder Hochschulzugänge migrantischer Studierender oder die Sichtbarkeit migrantischer Akteure in publikumszugewandten Berufen, wie bei der Deutschen Bahn oder an Flughäfen, aber auch in Nachrichtensendungen, Medien, Politik usw., verdeutlichen, dass strukturell und symbolisch mehr Teilhabe und Repräsentation erkennbar ist. Dabei geht es neben der Anerkennung von ungleichen Ausgangslagen und der Aushandlung von Antidiskriminierungsmaßnahmen zur Behebung von Ungleichheiten auch um die Aushandlung postmaterialistischer Anerkennungsebenen, wie Zugehörigkeit zur nationalen Erzählung oder zum deutschen »Wir«.

3. Gibt es postmigrantische Allianzen? Ja. Zunehmend alliieren sich Menschen entlang der Frage, wie sie in diverser werdenden Gesellschaften zusammenleben wollen. Dabei entstehen neue gesellschaftliche Lager jenseits der Migrations- und Herkunftsfrage. Das Spektrum ist sehr heterogen und umfasst Konservative, Liberale und Linke, Alte wie Junge, migrantische und nichtmigrantische Personen gleichermaßen entlang der Haltung, ob in Demokratien grundsätzlich allen Menschen die gleichen Rechte zukommen sollten. Die postmigrantischen Allianzen sind auch von der Erkenntnis geprägt, dass für die Umsetzung der Gleichheitsprämisse und der Teilhabegerechtigkeit etablierte Privilegien in Frage gestellt und mittelfristig abgebaut werden müssen und dass dafür systemrelevante Veränderungen und Ressourcenaufbau notwendig sind.

4. Sind die Antagonisten auch vielfältig? Ja. Auch hier gruppieren sie sich vor allem entlang einer Haltung gegenüber zentralen Normen der pluralen Demokratie: Gleichheit für alle oder vor allem für die Anhänger*innen des eigenen Kollektivs? Anerkennung von Differenz oder Abwehr von Vielfalt? Im antagonistischen Feld finden sich 
nicht nur Radikale, Islamisten oder Populisten, sondern auch jene, die mit Hybridität und Ambiguität hadern und sich nach klaren Ordnungsprinzipien sehnen, z. B. christlich Konservative, sozialdemokratische Mittelschicht und altfeministische Dogmatikerinnen. Das Spektrum der Antagonisten ist also ebenfalls sehr heterogen.

5. Welche Ambivalenzen sind sonst noch erkennbar? Die deutsche Gesellschaft ist ambivalent und vielstimmig. Auf der einen Seite werden Gleichheitsrechte garantiert, auf der anderen Seite ist die empirische Ungleichheit weiterhin präsent. Migrant*innen wird vorgeworfen, sich nicht mit Deutschland zu identifizieren - gleichzeitig werden sie als Fremde externalisiert. Integration wird erwartet, aber Aufstiege werden verwehrt. Es gibt eine Polarisierung entlang der Frage des Umgangs mit Pluralität. Die pmG verhandelt die Demokratiefrage entlang der Migrationsfrage. Migration dient dabei als Platzhalter für zentrale gesellschaftliche Debatten um Gleichheit, Anerkennung, Teilhabe - also die Versprechen der pluralen Demokratie.

Dieses Versprechen der pluralen Demokratie umzusetzen, das ist es, was die postmigrantische Gesellschaft in Aussicht stellt - das, was sie dynamisch antreibt, das, worum sie in ihren Aushandlungs- und Anerkennungsbewegungen kreist. Dass die Aushandlungen von Gleichheit, Anerkennung und Teilhabe zu Polarisierungen führen, weil sie Positionen und Privilegien hinterfragen und von manchen nur für die eigene soziale Gruppe beansprucht und nicht allen gleichermaßen zugestanden werden, ist in diesem Buch ausgiebig besprochen worden. Allerdings soll es nicht damit enden, diese Polarisierung als fatalistische Zwangsläufigkeit stehen zu lassen. Die Feministin und Rassismuskritikerin bell hooks schrieb schon 1994, »daß Personen mit vielen Privilegien, die in keiner Weise Opfer sind, sich aufgrund ihrer politischen Entscheidung für die Unterdrückten einsetzen können. Diese Solidarität muß nicht unbedingt auf gemeinsamen Erfahrung beruhen. Sie kann sich auf das politische und ethische Verständnis von Rassismus und die Absage an Dominanz gründen." (bell hooks 1994: 23) Sie ging davon aus, dass die Erziehung zu einem kritischen Bewusstsein auch jene Menschen, die direkt davon profitieren, in die Lage versetzen kann, sich der Herrschaftsstrukturen zu entledigen, in denen sie verwurzelt sind, ohne dass sie dies als Selbstaufgabe oder als Opfer sehen. 
Das Bewusstsein, dass das Versprechen der Gleichheit nicht nur ein universales und abstraktes ist, sondern auch ein nationales und konkretes und dass es eingefordert werden muss, nimmt in der postmigrantischen Gesellschaft zu. Es wird am Beispiel der Migrationsfrage verhandelt - aber nur exemplarisch. Dahinter stehen bereits vielschichtige Allianzen, die darüber hinausweisen: Indem sie die Migrationsfrage als einen Teil der Fragen um Gerechtigkeit neben anderen, wie z. B. Klima-, Feminismus-, Bildungs- oder Klassenfragen verhandeln, machen sie deutlich, dass eine postmigrantische Perspektive sich dem Versprechen der Gerechtigkeit zuwendet und nur gemeinsam und mit vielschichtigen Allianzen ausgehandelt und anerkannt werden kann. 
